\title{
Psychomotor control in a virtual laparoscopic surgery training environment: gaze control parameters differentiate novices from experts
}

\author{
Mark Wilson • John McGrath • Samuel Vine • \\ James Brewer · David Defriend $\cdot$ Richard Masters
}

Received: 6 November 2009/Accepted: 22 February 2010/Published online: 24 March 2010

(c) The Author(s) 2010. This article is published with open access at Springerlink.com

\begin{abstract}
Background Surgical simulation is increasingly used to facilitate the adoption of technical skills during surgical training. This study sought to determine if gaze control parameters could differentiate between the visual control of experienced and novice operators performing an eye-hand coordination task on a virtual reality laparoscopic surgical simulator (LAP Mentor ${ }^{\mathrm{TM}}$ ). Typically adopted hand movement metrics reflect only one half of the eye-hand coordination relationship; therefore, little is known about how hand movements are guided and controlled by vision. Methods A total of 14 right-handed surgeons were categorised as being either experienced (having led more than 70 laparoscopic procedures) or novice (having performed fewer than 10 procedures) operators. The eight experienced and six novice surgeons completed the eye-hand coordination task from the LAP Mentor basic skills package while wearing a gaze registration system. A variety of performance, movement, and gaze parameters were recorded and compared between groups.
\end{abstract}

M. Wilson $(\bowtie) \cdot S$. Vine

School of Sport and Health Sciences, University of Exeter,

St Luke's Campus, Exeter EX1 2LU, UK

e-mail: Mark.Wilson@exeter.ac.uk

J. McGrath

Department of Urology, Royal Devon and Exeter NHS

Foundation Trust, Exeter EX2 5DW, UK

J. Brewer · D. Defriend

Centre for Innovation and Training in Elective Care,

Torbay Hospital, Devon TQ2 7AA, UK

\section{R. Masters}

Institute of Human Performance, University of Hong Kong,

Pokfulam Road, Hong Kong, China
Results The experienced surgeons completed the task significantly more quickly than the novices, but only the economy of movement of the left tool differentiated skill level from the LAP Mentor parameters. Gaze analyses revealed that experienced surgeons spent significantly more time fixating the target locations than novices, who split their time between focusing on the targets and tracking the tools.

Conclusion The findings of the study provide support for the utility of assessing strategic gaze behaviour to better understand the way in which surgeons utilise visual information to plan and control tool movements in a virtual reality laparoscopic environment. It is hoped that by better understanding the limitations of the psychomotor system, effective gaze training programs may be developed.

Keywords Eye-hand coordination - Virtual reality . Gaze strategy $\cdot$ Psychomotor control $\cdot$ Laparoscopy training

Due to benefits in patient recovery, an increasing number of surgical procedures are being performed using laparoscopic techniques. Despite the advantages, laparoscopic surgery is technically demanding and requires new psychomotor skills that differ from those needed in conventional open surgery [1]. The difficulty in learning effective laparoscopic skills is compounded by other forces acting upon the surgical training environment. These issues include the requirement for greater efficiency in surgical training due to duty hour restrictions; the stringent financial reality of surgical training budgets; and the increasing public demand to demonstrate some level of competence prior to performing procedures in the operating room [2]. In this complex socioeconomic environment, virtual reality (VR) training simulators have been proposed to offer an 
effective way to train technical skills through repetitive practice in a safe, nonthreatening environment. Indeed, recent systematic reviews have demonstrated that such training can translate into improved outcomes in the operating room $[3,4]$.

An advantage of VR training over inanimate bench training methods is that objective performance assessment is provided immediately and without the need for monitored supervision [5]. However, although recent studies have started to support the discriminatory validity of these measures [1, 2, 6-8], it is still not entirely obvious as to how a trainee surgeon would use this feedback to guide subsequent learning: How does one train to improve "economy of movement" based on a percentage score? Performance will (likely) improve over time with continued experience with the training tasks, and the learner will be able to chart his/her improvement with this feedback. However, given the training pressures highlighted earlier, more attention needs to be applied to understanding how technical laparoscopy skills should optimally be learned through effective training curricula [6, 9-11].

Given this shortcoming, there is a need for a solid theoretical base for the effective teaching of technical (psychomotor) skills in surgery [12, 13]. Recent research examining the skilful performance of visually guided movements from other motor domains (e.g. driving and sport) has implicated the importance of gaze control in optimising subsequent motor control [14, 15]. In contrast, there has been little research focused on the strategic gaze behaviours of surgeons performing psychomotor, laparoscopic tasks. Indeed, the process measures that are examined are those from the "surgeon-tool" (S-T) interface (tool movement metrics) as opposed to the "surgeonmonitor" (S-M) interface (gaze metrics) [16].

The purpose of the current study is therefore to further our understanding of the process measures underlying expert performance in a VR laparoscopic eye-hand coordination task. In addition to comparing expert-novice differences in performance and process measures relating to the S-T interface (e.g. economy of movement), process measures relating to the $\mathrm{S}-\mathrm{M}$ interface are considered. The over-riding intention is to understand more about the eyehand coordination constraints in laparoscopic surgery so that the development of effective gaze training programmes for novice surgeons can be initiated.

\section{Methods}

\section{Participants}

A total of 14 surgeons volunteered to take part in the study (10 males, 4 females; mean age $=32.9$ years, range $=23-49$ years $)$. All participants were right-handdominant and were classified as novice or experienced laparoscopic surgeons according to the number of laparoscopic procedures they had led. Six novices ( 3 males, 3 females) had performed fewer than 10 procedures and eight experts ( 7 males, 1 female) had led more than 70 procedures (range $=70-700$ ). While this is acknowledged to be a relatively low sample size, power calculations using mean and standard deviations from previous studies using the same task and similar groupings [1, 7] suggested that these were appropriate group numbers for a one-tailed test with $\alpha=0.05$ and power $(1-\beta)=0.8$.

\section{Apparatus and task}

Testing took place on a LAP Mentor ${ }^{\mathrm{TM}}$ (Simbionix USA Corp., Cleveland, OH, USA) VR laparoscopic surgical simulator, based at the Centre for Innovation and Training in Elective Care, Torbay Hospital. The "eye-hand coordination" task from the basic skills training module was used for this study because previous research demonstrated that this task validly differentiates expert and novice surgeons $[1,7,17]$. To complete the task 10 flashing balls set at different heights and depths must be touched using one of two instruments, one held in each hand. One of the instruments is blue and the other is red and they become visible on the screen as soon as they are inserted. During the task flashing balls of each colour must be touched using the tip of the same colour instrument within a set time period. After one ball is touched (or time has run out), the next ball starts flashing and must be touched. Although the presentation of red and blue balls is pseudorandomised (e.g. there may be two blue balls in a row), each trial consists of five red and five blue balls.

Participants were fitted with an Applied Science Laboratories Mobile Eye gaze registration system (ASL, Bedford, MA; see Fig. 1), which measures eye-line of gaze using dark pupil tracking. The system incorporates a pair of lightweight glasses fitted with eye and scene cameras and a set of three LEDs which project harmless near-infrared (IR) light onto the eye. The near-IR light is not visible to the user so it does not cause a distraction; however, it is visible to the eye camera. Some of this light is reflected by the cornea (corneal reflection) and appears to the eye camera as a triangle of three dots at a fixed distance from each other (Fig. 2). The pupil appears black as light does not exit the inside of the eye, enabling the system to register its position and determine its center (the magenta circle in Fig. 2). When the eye turns, the center of the pupil moves relative to the head; however, the corneal reflection remains in the same position. Therefore, by comparing the vector (angle and distance) between the pupil and the 


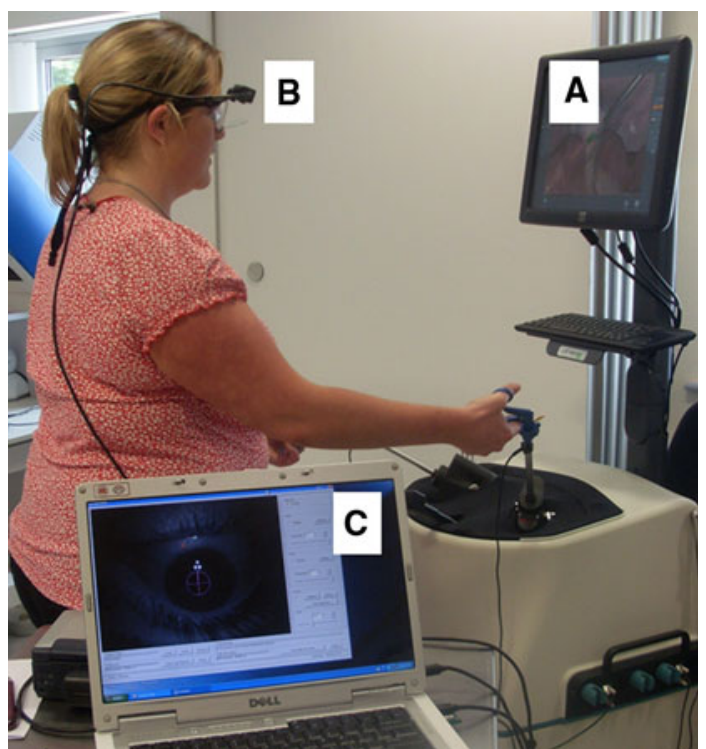

Fig. 1 The testing environment demonstrating the LAP Mentor virtual reality simulator (A), the lightweight optics unit $(\mathbf{B})$, and the supporting software running on the laptop $(\mathbf{C})$

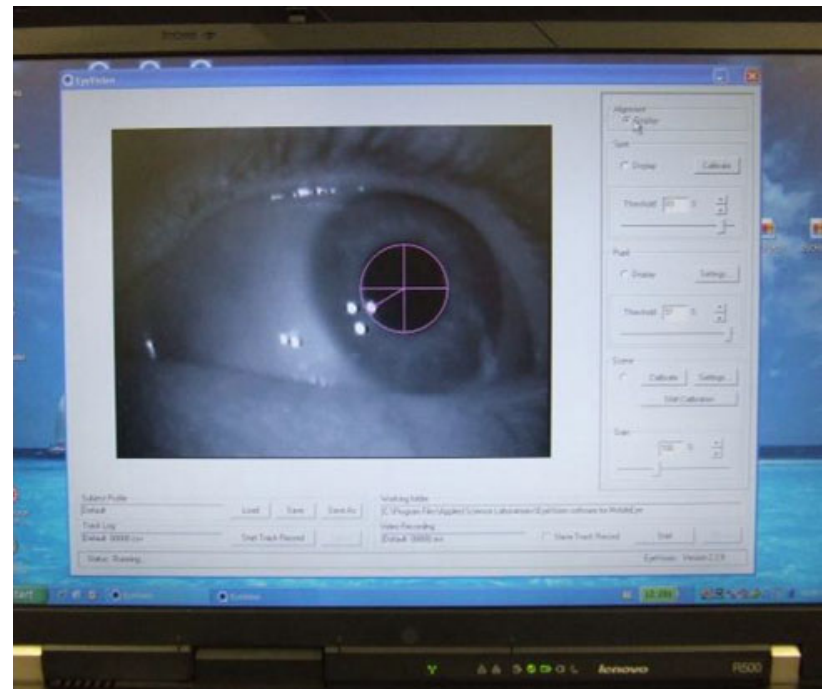

Fig. 2 An image from the eye camera in the Eyevision software environment showing the corneal reflection from the LEDs (three white dots); the pupil center (midpoint of the magenta circle) and the vector line between the pupil centre and the corneal reflection

cornea, the eye-tracking system can compute the angle that the eye is pointed (Fig. 2).

The system also incorporates a recording device (a modified digital video cassette recorder) that combines the two video streams from the eye and scene cameras at $25 \mathrm{~Hz}$. The recorder is attached to a laptop installed with Eyevision (ASL) software; both were placed on a table to the side of the participant (Fig. 1). By teaching the system how the angles calculated by the eye camera relate to the

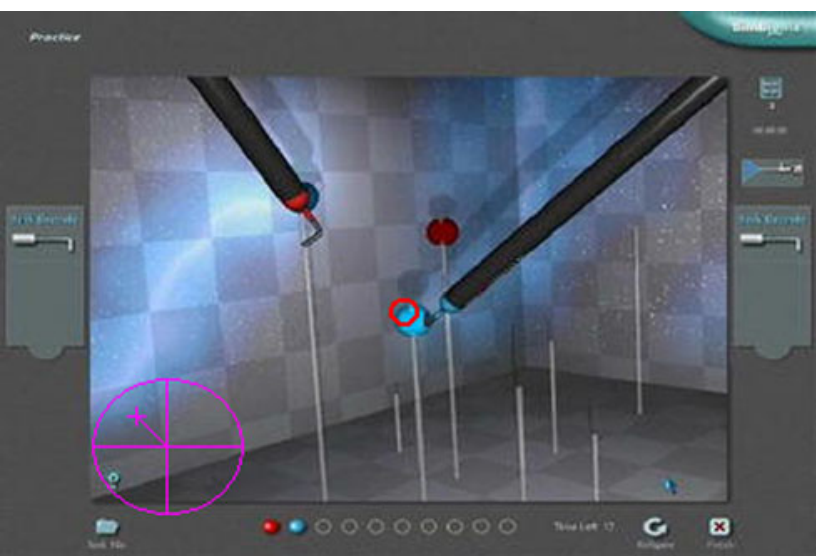

Fig. 3 The eye-hand coordination task showing the target balls, the "virtual" instruments, the gaze cursor (on the central flashing ball), and the vector angle calculation (bottom left of screen)

image from the second camera that is viewing the environment (the scene camera), the eye tracker can compute what the eye is pointed at. A circular cursor, representing $1^{\circ}$ of visual angle with a $4.5-\mathrm{mm}$ lens, indicating the location of gaze in a video image of the scene (spatial accuracy of $\pm 0.5^{\circ}$ visual angle; $0.1^{\circ}$ precision), is viewed in real time and recorded for subsequent offline analyses. Figure 3 is a representative image of a frame of video data from the Eyevision software environment showing the momentary point of gaze (the small red circle located on the target ball).

\section{Procedure}

Participants arrived at the Training Centre individually at prearranged times. They first read an information sheet describing the aims of the study, before completing a demographic questionnaire and providing written informed consent to participation. Participants were fitted with the eye tracker which was then calibrated using six visual landmarks on the LAP Mentor display screen. They then performed three consecutive attempts at the eye-hand coordination task, as part of a series of activities, before being debriefed and thanked for their participation in the study.

\section{Measures}

\section{Performance}

Performance was assessed in terms of accuracy (number of balls hit) and speed (task completion time).

\section{Tool movement process measures}

The number of movements (NOM) and economy of movement (EOM) parameters for each tool were selected 
from the LAP Mentor parameter options to reflect effective and efficient tool control. A new movement is defined as either continuous movement of the tool for more than $3 \mathrm{~mm}$ or a change in direction of $90^{\circ}$. EOM is defined as the ratio of the shortest path length after the ball starts flashing to the actual path length after the ball starts flashing (presented as a percentage).

\section{Gaze process measures}

Two categories of gaze measures were determined to reflect the efficiency of psychomotor control. First, fixation rate was calculated for each trial as a general measure of gaze strategy, with more efficient gaze behaviour indexed by lower fixation rates (i.e. fewer and longer fixations). Fixation rate was operationally defined as the number of fixations made per second per trial. Second, the percentage of time spent fixating on one of the two critical locations throughout the trial was calculated to provide more information regarding what the participants attended to. Previous research has demonstrated that experts are more likely to fixate on the target (i.e. the flashing ball in this study) while novices are more likely to track the tool as it moves toward the target [18]. A fixation was defined as a gaze of long enough duration to allow information processing ( $\geq 120 \mathrm{~ms}$ ) to a single location (within $1^{\circ}$ visual angle).

\section{Analysis}

The first trial was considered a familiarization attempt for all participants, providing insight into the testing protocol while limiting additional learning opportunities prior to testing. Data from the two subsequent trials were averaged to provide a mean value for each variable for each participant to be used for subsequent analyses. Performance and tool process measures were downloaded directly from the LAP Mentor software environment after each trial. The gaze data were analysed in a frame-by-frame manner (40 frames for $1 \mathrm{~s}$ of video) using GazeTracker (Eye Response Technologies, Charlottesville, VA, USA) video analysis software. For each individual ball-touch attempt, areas of interest ("Lookzones") were created and maintained around the target ball and the relevant instrument as the video progressed. The software then automatically provided summary gaze data and gaze data relevant to each area of interest for the trial as a whole. The researcher analysing the gaze data was experienced in performing such analyses and blind to the skill levels of the participants to protect against analysis bias.

Shapiro-Wilk tests revealed that all data were normally distributed, apart from performance accuracy data. Group differences in performance accuracy were therefore analysed using the nonparametric Mann-Whitney $U$ test.
Differences between task completion time, EOM, NOM and fixation rate for each group were analysed using a series of independent group $t$ tests. Differences in the locations (tools or ball) fixated upon were subjected to a mixed-design $2 \times 2$ ANOVA (group $\times$ location), with post-hoc $t$ tests used to follow up significant interaction effects. All analyses were performed using SPSS 15.0 for Windows (SPSS Inc., Chicago, IL, USA).

\section{Results}

Performance measures

There was no significant difference in accuracy between the experienced and novice surgeons $(Z=0.53$, $P=0.58$ ); however, experienced surgeons completed the task significantly faster than their novice counterparts $\left(t_{12}=2.64, P<0.05\right)$. Performance measures are presented in Table 1.

\section{$\mathrm{S}-\mathrm{T}$ process measures}

There were no significant differences in the number of movements (NOM) made by either group of surgeon for the left $\left(t_{12}=1.18, P=0.26\right)$ or right $\left(t_{12}=1.76\right.$, $P=0.10)$ tool. There was also no significant difference in the economy of movement (EOM) of the right tool ( $\left.t_{12}=1.06, P=0.31\right)$ between the surgeons. However, experienced surgeons had significantly higher economy of movement scores for the left-hand tool than their novice counterparts $\left(t_{12}=2.38, P<0.05\right)$. $\mathrm{S}-\mathrm{T}$ process measures are presented in Table 1.

\section{S-M process measures}

There were no significant differences in the fixation rates between experienced and novice surgeons $\left(t_{12}=0.69\right.$, $P=0.50)$. The ANOVA of the percentage time spent fixating on each gaze location revealed a significant main

Table 1 Mean $( \pm \mathrm{SD})$ performance and process measures for novice and experienced groups

\begin{tabular}{lcc}
\hline Parameter & Novice & Experienced \\
\hline Accuracy (/10) & $9.50 \pm 0.45$ & $9.63 \pm 0.44$ \\
Completion time (s) & $74.50 \pm 13.44$ & $56.56 \pm 11.93$ \\
NOM (R) & $24.67 \pm 8.13$ & $18.80 \pm 4.07$ \\
NOM (L) & $26.83 \pm 7.91$ & $22.59 \pm 5.61$ \\
EOM (R) (\%) & $53.12 \pm 15.93$ & $59.67 \pm 6.61$ \\
EOM (L) (\%) & $45.64 \pm 9.38$ & $62.19 \pm 14.88$ \\
Fixation rate (/second) & $1.85 \pm 0.14$ & $1.90 \pm 0.16$ \\
\hline
\end{tabular}




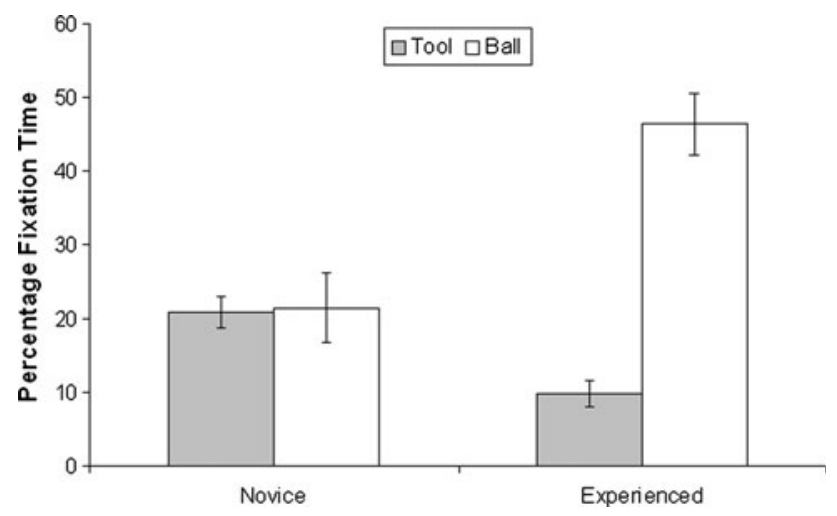

Fig. 4 The percentage of total fixation duration to each of the two relevant locations for novice and experienced surgeons $( \pm$ SEM)

effect for location $\left(F_{1,12}=34.43, P<0.001\right)$ and no significant main effect for ability level $\left(F_{1,12}=3.53\right.$, $P=0.09)$. These results were qualified by a significant interaction effect $\left(F_{1,12}=32.21, P<0.001\right)$. As Fig. 4 demonstrates, experts spent significantly more time fixating on the target ball than their novice counterparts $(P<0.001)$, while novices spent significantly more time tracking the tools than their expert counterparts $(P<0.001)$. Novices spent similar amounts of time fixating on the target ball and tracking the tools, while experts spent significantly more time fixating on the target balls compared to tool tracking $(P<0.001)$.

\section{Discussion}

The aim of this research was to adopt a motor control perspective to the understanding of the eye-hand coordination advantage of experienced laparoscopic operators. While recent studies have supported the construct validity of the eye-hand coordination task from the LAP Mentor training package $[1,7,17]$, there has yet to be an analysis of the gaze strategy used by operators on this task. Current research has focused exclusively on tool measures and on how the hand moves to complete the task. Accordingly, researchers are considering only part of the eye-hand coordination relationship and are potentially ignoring important information related to the sources of information used to guide subsequent tool movements. We demonstrate that gaze data can discriminate between levels of experience and suggest that this may provide useful information by which to guide basic laparoscopic skill development.

The performance results demonstrated that experienced laparoscopic surgeons were significantly faster in completing the task than their novice counterparts, although there were no differences in their level of accuracy. An accuracy measure assessing only the number of target balls touched is rather insensitive and it is perhaps not surprising that a ceiling effect was found for this relatively straightforward task. Indeed, previous researchers have argued that completion time is a better measure of the construct validity of the task [1,7]. Completion times in the current study were of similar magnitude to those of Yamaguchi et al. [1] (novices, $73.4 \mathrm{~s}$; experts, $52.6 \mathrm{~s}$ ) and Andreatta et al. [7] (novices, $72.3 \mathrm{~s}$; experts, $51.0 \mathrm{~s}$ ), providing further support for the discriminatory power of the completion time measure.

To better understand the mechanisms underlying this performance advantage, it is necessary to examine the process measures that also discriminate between experience levels. These measures are important, because by training novices to improve these critical components of their laparoscopic technique, performance should also be improved. Our results demonstrated that the economy of movement (EOM) of the left (nondominant) hand was able to significantly differentiate between experienced and novice operators. Yamaguchi et al. [1] also found that nondominant-hand performance was more sensitive than dominant-hand performance for this relatively simple task, suggesting that novices should concentrate on the nondominant hand to expedite skill learning. However, while such nondominant-hand EOM data may be useful in setting criteria-based training targets [6,7], it is not so useful in guiding learners how to improve. This appears to be a common weakness in many criteria-based training protocols.

The current research extended previous tests of construct validity by considering process measures beyond those provided by the VR simulator itself to include eye movement and gaze parameters. While the importance of strategic gaze behaviour in optimising accurate motor control has been demonstrated reliably in tasks as varied as pointing [19], reaching and grasping [20], driving [14, 21], sport skills [22] and even sandwich-making [23], there has been little research focused on the strategic gaze behaviours of surgeons performing laparoscopic tasks. The current findings suggest that it is not the efficiency of the eye movements in general that is important, but the relevance of the target in optimally controlling the ensuing motor action.

There were no significant differences in the fixation rate (the number of fixations made in a given time period) between experienced and less experienced participants in this study (Table 1). This result differs from that of Kocak et al. [16], who found that surgeons with greater experience tended to move their eyes less and spend more time fixed on a given point. This difference in results may be due to differences in the eye-tracking equipment used, as Kocak et al. used a device that measured only eye movements as opposed to the more detailed gaze analyses carried out in the current study. Indeed, while we found that the general 
eye movement rates of experts were similar to those of novices, the locations (targets) of the interceding fixations were significantly different. Experts spent more time using a "target-focused" gaze strategy as opposed to a "toolfollowing" strategy. Novices focused equally on both locations, in what Law et al. [18] termed a "switching strategy" (Fig. 4).

So why might a target-focused strategy underpin more effective tool movement control and outcome performance in laparoscopic skill training? Research from the neuroscience and motor control literature suggests that eye movements and the gaze system that controls their location play a key role in coordinating precision motor actions. Indeed, the neural mechanisms regulating goal-directed movements actually profit from the accurate and timely spatial information of the foveated target [20, 24]. While experts are able to maintain focus on the target and locate the position of their tools with peripheral vision, novices appear to switch between tool and target position in order to determine the relative position of both. This strategy not only disrupts effective psychomotor coordination, but will likely impair the processing of important visual information required for decision-making in more complex laparoscopic tasks.

The implications of the current research go beyond providing further objective criteria by which to differentiate expert and novice performance. Instead, the research may help to initiate interest in examining how surgical trainers may optimise the laparoscopic training environment [25]. There has been recent interest in promoting partnerships with experts in motor learning, sport psychology and kinesiology in order to apply theoretically rigorous motor learning frameworks to surgical training [26, 27]. For example, Masters et al. [28] have recently applied an observational learning protocol to teaching surgical skills, with positive results. Recent findings from the sport literature might also suggest that gaze-training protocols could be successfully applied to laparoscopic surgery. For example, not only did novice golfers who followed a gaze-focused training protocol learn more quickly than those following a movement-focused training protocol, but they were more resistant to stress effects in a subsequent transfer test [29].

To conclude, the current study provided additional support for the construct validity of the LAP Mentor eyehand coordination task and for the use of strategic gaze measures to differentiate between levels of experience in laparoscopic procedures. The results have also started to illuminate how surgeons utilise visual information to plan and control tool movements in a virtual reality laparoscopic environment. Importantly, evidence from sport tasks would suggest that feedback about gaze behaviour may be effective for training purposes and might help the expedient development of robust technical skills.

Acknowledgments This work was supported by a bilateral research grant from the Economic and Social Research Council, UK, and the Research Grants Council, Hong Kong (RES-000-22-3016), awarded to the first and last authors.

Disclosures Drs. Wilson and Brewer, Mr. Vine, Mr. McGrath, Mr. Defriend and Professor Masters have no conflicts of interest or financial ties to disclose.

Open Access This article is distributed under the terms of the Creative Commons Attribution Noncommercial License which permits any noncommercial use, distribution, and reproduction in any medium, provided the original author(s) and source are credited.

\section{References}

1. Yamaguchi S, Konishi K, Yasunaga T, Yoshida D, Kinjo N, Kobayashi K, Ieiri S, Okazaki K, Nakashima H, Tanoue K, Maehara Y, Hashizume M (2007) Construct validity for eye-hand coordination skill on a virtual reality laparoscopic surgical simulator. Surg Endosc 21:2253-2257

2. Andreatta PB, Woodrum DT, Birkmeyer JD, Yellamanchilli RK, Doherty GM, Gauger PG, Minter RM (2006) Laparoscopic skills are improved with LapMentor ${ }^{\mathrm{TM}}$ training: results of a randomized, double-blinded study. Ann Surg 243:854-863

3. Dunkin B, Adrales DL, Apelgren K, Mellinger JD (2007) Surgical simulation: a current review. Surg Endosc 21:357-366

4. Gurusamy K, Aggarwal R, Palanivelu L, Davidson BR (2008) Systematic review of randomized controlled trials on the effectiveness of virtual reality training for laparoscopic surgery. Br J Surg 95:1088-1097

5. Avodeji ID, Schijven M, Jakimowicz J, Greve JW (2007) Face validation of the Simbionix LAP Mentor virtual reality training module and its applicability in the surgical curriculum. Surg Endosc 21:1641-1649

6. Aggarwal R, Crochet A, Dias A, Misra A, Ziprin P, Darzi A (2009) Development of a virtual reality training curriculum for laparoscopic cholecystectomy. Br J Surg 96:1086-1093

7. Andreatta PB, Woodrum DT, Gauger PG, Minter RM (2008) LapMentor $^{\mathrm{TM}}$ metrics possess limited construct validity. Simul Healthc 3:16-25

8. Zhang A, Hünerbein M, Dai Y, Schlag PM, Beller S (2008) Construct validity testing of a laparoscopic surgery simulator (Lap Mentor): evaluation of surgical skill with a virtual laparoscopic training simulator. Surg Endosc 22:1440-1444

9. Boehler ML, Schwind CJ, Rogers DA, Ketchum J, O'Sullivan E, Mayforth R, Quin J, Wohltman C, Johnson C, Williams RG, Dunnington G (2007) A theory-based curriculum for enhancing surgical skillfulness. J Am Coll Surg 205:492-497

10. Stefanidis D, Korndorffer JR Jr, Heniford BT, Scott DJ (2007) Limited feedback and video tutorials optimize learning and resource utilization during laparoscopic simulator training. Surgery 142:202-206

11. Verdaasdonk EG, Dankelman J, Lange JF, Stassen LP (2008) Incorporation of proficiency criteria for basic laparoscopic skills training: how does it work? Surg Endosc 22:2609-2615

12. Hamdorf J, Hall JC (2000) Acquiring surgical skills. Br J Surg $86: 28-37$ 
13. Wong JA, Matsumoto ED (2008) Primer: cognitive motor learning for teaching surgical skill-how are surgical skills taught and assessed? Nat Clin Pract Urol 5:47-54

14. Wilson M, Chattington M, Marple-Horvat DE (2008) Eye movements drive steering: the effect of reduced eye movement distribution on steering and driving performance. J Mot Behav 40:190-202

15. Wilson MR, Vine SJ, Wood G (2009) The influence of anxiety on visual attentional control in basketball free-throw shooting. J Sport Exerc Psychol 31:152-168

16. Kocak E, Ober J, Berme N, Melvin WS (2005) Eye motion parameters correlate with level of experience in video-assisted surgery: objective testing of three tasks. J Laparoendosc Adv Surg Tech 15:575-580

17. McDougall EM, Corica FA, Boker JR, Sala LG, Stoliar G, Borin JF, Chu FT, Clayman RV (2006) Construct validity testing of a laparoscopic surgical simulator. J Am Coll Surg 202:779-787

18. Law B, Atkins MS, Kirkpatrick AE, Lomax AJ, Mackenzie CL (2004) Eye gaze patterns differentiate novice and experts in a virtual laparoscopic surgery training environment. In: Duchowski AT, Vertegaal R (eds) Proceedings of the eye tracking research and application symposium, San Antonio, TX, pp 41-47

19. Sarlegna F, Blouin J, Bresciani JP, Bourdin C, Vercher JL, Gauthier GM (2003) Target and hand position information in the online control of goal-directed arm movements. Exp Brain Res 151:524-535

20. Neggers SF, Bekkering H (2000) Coordinated control of eye and hand movements in dynamic reaching. Hum Mov Sci 21:349-376
21. Wilson M, Stephenson S, Chattington M, Marple-Horvat DE (2007) Eye movements coordinated with steering benefit performance even when vision is denied. Exp Brain Res 176:397412

22. Vickers JN (2007) Perception, cognition and decision training: the quiet eye in action. Human Kinetics, Champaign, IL

23. Hayhoe MM, Shrivastava A, Mruczek R, Pelz JB (2003) Visual memory and motor planning in a natural task. J Vis 3:49-63

24. Neggers SF, Bekkering H (2001) Gaze anchoring to a pointing target is present during the entire pointing movement and is driven by a non-visual signal. J Neurophysiol 86:961-970

25. Wilson M, McGrath J, Coleman M (2010) Developing basic eyehand coordination skills for laparoscopic surgery using gaze training. BJU Int. doi:10.1111/j.1464-410X.2010.09249.x

26. Mendoza KA, Hauge LS, DaRosa DA (2004) The responsibilities and contributions of professional educators in surgery departments. Am J Surg 188:126-130

27. Thuraisingam AI, Levine DF, Anderson JT (2006) Can research in sports and other motor skills help improve endoscopy training? Gastrointest Endosc 63:276-279

28. Masters RS, Lo CY, Maxwell JP, Patil NG (2008) Implicit motor learning in surgery: implications for multi-tasking. Surgery 143:140-145

29. Vine SJ, Wilson MR (2009) Quiet eye training improves performance and protects against stress in a golf putting task. British association of sport and exercise science annual conference book of abstracts, p S150 